\title{
Geometric error analysis in applied calculus problem solving
}

\author{
Ahmed Ibrahim Usman \\ Department of General studies (Mathematics), Jubail University College, Jubail Industrial City, Kingdom of Saudi Arabia \\ For correspondence: usmana@ucj.edu.sa
}

\begin{abstract}
The paper investigates geometric errors students made as they tried to use their basic geometric knowledge in the solution of the Applied Calculus Optimization Problem (ACOP). Inaccuracies related to the drawing of geometric diagrams (visualization skills) and those associated with the application of basic differentiation concepts into ACOP solution were reported. A test instrument was used to collect quantitative data, while qualitative data were generated using follow- up interviews (stimulated recall). The targeted samples were freshmen students who registered for Calculus I in the department of Mathematics at a University in south eastern region of the United States of America, USA. The study indicated that students had achieved a very low success rate on the ACOP solution process, immediately after receiving/completing instruction on the optimization in their calculus I class. In general, they failed to integrate basic geometric competences required in the ACOP solution. Qualitative evidence from students' test performance indicated that failure to visualize geometric diagrams from word problems tended to preclude them getting the required formula. The overall finding of the research was that students face structural and procedural setbacks that ultimately led to a worsening of the ACOP solution process.
\end{abstract}

Keywords: applied calculus optimization problem, visualization, geometric, stimulated recall, and optimization.

\section{Introduction}

Calculus is a useful area of Mathematics whose application cuts across many disciplines; its worthiness was equally elaborated by NCTM's, Curriculum and Evaluation Standards for Schools Mathematics (2000), as well as MAA/NCTM Joint Position Statement, (2012). Despite the importance of calculus, certain difficulties inhibit students from learning it, leading to unprecedented failure (Burton, 1989; Tall, 1992; Matta, Feinberg, Cardetti, 2012, Brijlall, 2013; Muzangwa \& Chifamba, 2012; Yasin\&Enver, 2007). The challenges faced by students taking Calculus at college level requires mastery of tools cutting across the mastery of algebra, geometry, trigonometry and coordinate geometry, MAA/NCTM position. Calculus taken during the first year in college clearly depends on students' actual mathematical skills that include algebra and geometry. Further calculus learning impediments, Usman (2008), Davis (1986), Ferrini-Mundy and Graham (1991), are that students resort to memorizing calculus solution skills without paying more attention to in-depth comprehension of core concepts. An effective platform to investigate problems associated with calculus learning that are grounded geometrically is applied calculus optimization problems (ACOP). ACOP is the set of word problems which requires the application of algebra, geometry, and basic differentiation skills from calculus to compute, interpret and analyze larger and smaller values of a model on some interval, and determine where the largest or smallest value occurs, (Usman, 2008).

\section{The Study}

\section{Purpose}

Basic knowledge of geometry is an essential tool for solving Calculus problem, MAA/NCTM position statement, 2012. The purpose of this sequential explanatory mixed methods study in two-phases 
would be to collect and analyze data on geometric errors committed in the process of ACOP solution quantitatively using descriptive statistics and then follow-up with qualitative analysis (interview transcription and thematic content analysis of the test manuscript), Creswell (2003). The descriptive statistics explains the characteristics of the population on visualization ability, labeling of geometric diagrams, matching of basic geometric shapes with their equivalent formula, as well as ability to solve ACOP completely. In a similar vein, the qualitative component using content analysis would further elucidate more on these errors in a more detailed fashion, Onwuegbuzie \& Teddlie (2003). The following Research questions were used for the study:

1. What kind of visualization ability do students possess in constructing geometric diagrams described in words?

2. Are students able to label geometric diagrams appropriately/inappropriately using variables?

3. Are students able to associate geometric diagrams with their corresponding algebraic equation(s)/formula(s)?

4. What kind of geometric ability do students possess in solving ACOP completely?

\section{Related Literature}

Visualization ability and knowledge of basic geometric diagrams are important in the solution process of many mathematical problems (particularly word problems) in applied calculus. The key concepts reviewed below are visualization and geometric learning difficulties. The overall purpose is to unveil the difficulties that students encounter in learning, understanding and applying these concepts.

\section{Visualization}

Mathematics education researchers earlier defined visualization, (Hershkowitz, 1990; Zimmermann \& Cunningham, 1991; Arcavi, 2002), and the focus of their definitions was centered on the ability to interpret diagrammatically what is in the mind. Many researches in mathematics education and other areas such as psychology, engineering, art etc., have been conducted on visualization. In many, the attempt was to show the importance and role of visualization in interpretation and applications, Arcavi; Dreyfus (1991); and Usman. In a study investigating the presence, role, extent and constraint of visual thinking in the problem solving processes, Presmeg \& Balderas-Cañas (n.d) reported that visualization may play a useful role in the problem solving strategies. In a related development, Stylianou \& Pitta-Pantazi (2002) investigated the patterns or characteristics of visual representation use that underlie successful problem solving. They identified some patterns of successful visualization which depends on "the individual's ability to select what needs to be visualized and what to be talked about in the mind; and the oscillation of the visualized and verbalized components and how these complement each other".

It was reported that understanding fundamental calculus concepts (e.g., limits, derivatives and integrals, NCTM) requires the use of visual representations. The ability to successfully solve many problems with calculus depends on visual images in the form of diagrams or graphs (Zimmerman \& Cunningham, 1991; Bremigan, 2005). Prerequisites identified in the work of Bremigan for visual thinking in calculus (applied calculus) include the ability to extract specific information from diagrams, an understanding of algebra (variables, equations, formulas) and geometry (plane and solid) as alternative languages for the expression of mathematical ideas, and knowledge of the rules and conventions associated with mathematical graphs. More often, calculus teachers assume that their students have these prerequisites skills and that students appreciate the important role of reasoning with visual representations, (Bremigan). It turns out that these assumptions are false when applied to real classroom situations. 
In an attempt to handle the issues raised by Bremigan above, earlier, Dreyfus (1991), called for paying more attention to visualization and visual reasoning in the teaching of mathematics, Gutiérrez (1997). Dreyfus clearly stated that "visual reasoning in mathematics is important in its own right and that therefore we need to develop and give full status to purely visual mathematics activities", (p.46). To further consolidate the proposal of Dreyfus, Gutiérrez outlined a model of using visualization (mental images, external representations, processes, and abilities of visualization) as a component of the teaching, learning or reasoning in mathematics classrooms.

\section{Geometric Learning}

Teaching and learning of geometry has been a subject of research for many decades. Issues cutting across geometric curricula received a lot of attention, more specifically, less attention was given to geometry compared to algebra over the years, (Jones, 2000; 2002; Jones \& Mooney, 2003 ;), cited by Hohenwarter \& Jones (2007). Students' poor performance in geometric tasks in different countries across the globe was also an area that occupied the mathematics education research community for some time, for example, see Battista (2007; 2001a); Martin \&Structchens (2000); Clements (2003); Clement et al (1997); Usman; Chappell \& Thompson (1999); Nunes, Light, \& Mason (1993); Pesek \& Kirshner (2000); and Woodward \& Byrd (1983), for additional information.

Moreover, another theme added to the research in mathematics education was students' geometrical reasoning (knowledge, abilities, strategies, difficulties) Panaoura, \& Gagatsis, (2009). Koc, Y, et. al, (2012) investigated students' performance in geometrical structures (identifying, naming, and drawing of geometrical objects on a dot paper), it was revealed that students had difficulty thinking in three dimensional space. Moreover, the study also discovered that their van Hiele level for three dimensional spaces could not be trace. Similarly, in an experiment conducted by Marchett, $\mathrm{P}$, et al. (2005), exploring student's concepts of perimeter and area, it was shown that there exist difficulties in dealing with them.

In a study investigating students' thinking in three-dimensional geometry, specifically, threedimensional objects and their properties, Pittalis \& Christou, (2010) reported that there is close relation between the representation reasoning and the mathematical properties reasoning, similarly between figural and conceptual features of three-dimensional geometry.

Looking at the abovementioned challenges and difficulties of geometric learning as well as utilization of visualization skills in solving mathematics problems, this paper reports how specific knowledge of basic geometric concepts, and visualization abilities facilitate or abate the solution of applied calculus problem solving.

\section{Method}

\section{Design}

This study applies a mixed methods research design. Specifically, it employs explanatory sequential mixed method design (Creswell, 2011). The design involves collecting data quantitatively (using a test instrument) and qualitatively (conducting clinical interviews, i.e. stimulated recall) sequentially. The two methods, quantitative and qualitative are used to detect errors and misconceptions with respect to the drawing of diagrams and visualization of solution of ACOP. Moreover, errors related to the use of basic differentiation skills were noted.

\section{Participants}

The target population was freshmen students taking calculus I in the Department of Mathematics, at a University in south eastern region of the USA. The portion of calculus I which was used for the study was applied calculus chapter, specifically, optimization problem. The test was given to them 
immediately after they completed that portion of the chapter. The total enrolment for Calculus I was one thousand and forty students in spring semester of 2008. A sample size of one hundred fifty-four students or approximately fifteen per cent of the total enrolment was used. Six sections out of twenty five are used for the study. The letters $(\mathrm{S}, \mathrm{J}, \mathrm{W}, \mathrm{T}, \mathrm{L}$, and $\mathrm{V}$ ) were the first initials of teachers who taught the sections. They were used to denote different sections that participate in the study.

\section{Instrument}

Quantitative data were collected using test instrument. The instrument consists of routine and nonroutine questions from basic geometry and applied calculus (optimization). They are carefully selected and design by the researcher to meet the objective of the study. To quantify the test results, a rubric was developed assigning scores for each question. A score of 0 to 9 points was allocated for questions 1, 2, 4 and 0 to 6 points for question 3. Questions 1, 2 and 4 had three sub-items and 3 points were allocated for each sub-item, whereas question 3 had six sub-items and each attract a score of one point. The data generated was interpreted using descriptive statistics (frequencies, mean, standard deviation, minimum and maximum).

\section{Stimulated recall}

For the purpose of selecting participants for the stimulated recall interviews, a simple random and convenient sampling technique were used (sequential mixed method design). A total of fifteen (15) interviews were conducted and each lasting for an average of forty (40) minutes. For each interviewee, an interview protocol was prepared before the interview based on the participants' test script. Each protocol contained hypotheses and probes on the student's performance from the test. The interviews were transcribed and analyzed, giving a broader perspective of students thought processes as revealed by them.

\section{Analysis}

The demographic characteristics of the sample are contain in Table 1 below. It shows frequencies and percentages of male and female participants, ninety-two (59.40\%), sixty-two (40.60\%) respectively. Moreover, the table also gave racial descriptions of the participants White, One hundred fourteen (73.50\%); Africa-America, twenty-two (14.20\%); and Other, fifteen $(9.70 \%)$. The analysis does not take into consideration different categories (i.e. gender or race), but rather, the approach was based on the population as a whole. The reliability test for the data collected is Cronbach's Alpha of $0.767(\alpha=$ 0.767), which is acceptable. According to George \& Mallery (2005), "the Cronbach's alpha is designed as a measure of internal consistency; that is, do all items within the instrument measure the same thing?"

Table 1: Frequencies and Percentages for Gender and Race

\begin{tabular}{|c|c|c|}
\hline Gender/Race & Frequencies & Percentages \\
\hline Male & 92 & $59.40 \%$ \\
\hline Female & 62 & $40.60 \%$ \\
\hline White & 114 & $74.03 \%$ \\
\hline African-American & 22 & $14.29 \%$ \\
\hline Other & 15 & $9.74 \%$ \\
\hline
\end{tabular}

\section{Quantitative and Qualitative Data Analysis}

Research question 1: What kind of visualization ability do students possess in constructing geometric diagrams described in words?

The focus of these research questions was to measure students' knowledge of basic geometric shapes as well as their application to physical situations. It also takes into account students' visualization 
ability and how it was incorporated into basic geometric shapes, such as rectangle, triangle, circle, cylinder, and so on. The visualization capability is expected to be used fluently. Three test items were designed by the researcher to assess these attributes of the first research question. The concepts assessed by the test items in this section include distinguishing a square from a rectangle as well as locating the squares that are supposed to be cut-off from the rectangular diagram; visualizing a cylinder inside a hemisphere; and understanding the concepts of cylinder, hemisphere, and inscription. Other anticipated conceptual difficulties comprise visualizing a movement from ordinary plane paper (two-dimensional) to solid (three-dimensional). Results from the quantitative portion of the test (see table 2) below indicated that the average score is 6.23 out of maximum of 9 possible points. The result also shows that 70 students $(45.8 \%)$ scored between 0 to 6 ; whereas 84 students (54.2\%) scored between 7 to 9 points.

Table 2: Descriptive Statistics of all Variables and Subjects.

\begin{tabular}{ccccccc}
\hline Variables & Mean & Mode & $\begin{array}{c}\text { Std. } \\
\text { Deviation }\end{array}$ & Minimum & Maximum & $\begin{array}{c}\text { Maximum } \\
\text { possible }\end{array}$ \\
\hline Diagcons* $^{*}$ & 6.23 & 7 & 2.04 & 0 & 9 & 9 \\
Labeldiag* $^{*}$ & 6.34 & 7 & 1.5 & 1 & 9 & 9 \\
Assdiag* $^{*}$ & 4.7 & 6 & 1.41 & 1 & 6 & 6 \\
Optpbm* $^{*}$ & 1.83 & 2 & 1.41 & 0 & 6 & 9 \\
\hline
\end{tabular}

*Diagcons: Diagram construction, Labeldiag: Label a Diagram, Assodiag: Associating a diagram with formulas, Optpbm: Optimization problem (full ACOP).

From the indicated quantitative outcome, the sample can be divided into two categories: those who scored between 0 to 6 points, and 7 to 9 points, using mean score of 6.23 as a bench-mark. In the $54.2 \%$ category of the sample in this isolated task, it was revealed that they performed minimal mistakes in their solution process. The isolated task is one that requires students to represent geometric word problems diagrammatically. A qualitative illustration of a representative sample from each group, ( $54.2 \%$ and $45.8 \%$ ), is given below. Subject $\mathrm{W}-2$ belongs to $54.2 \%$ category and has demonstrated an ability to visualize the geometric word problem fluently as well as in writing. (W-represents the section of the calculus I selected to take the test and 2 represents a second student selected randomly and conveniently who agreed to do the interview from $\mathrm{W}$-section). Below is an account of our interaction:

RCH: I want to know how you interpret the question.

W-2: You have cardboard box and in making it (demonstrating with hand how to fold it).

$\mathrm{RCH}$ : So you visualize it before you write?

W-2: Yeah

$\mathrm{RCH}$ : How about the second one?

W-2: $\quad$ For this one I wasn't quite sure how to do the top...kind of a cut-off. The visual representation doesn't need to be perfect. But again, you just need to visualize, because it is purely conceptual.

W-2's test script and verbal demonstration have indicated an understanding of the relationship between concept definition and concept image (Hershkowitz) of a rectangular box. The interviewee clearly describes a rectangular box when the four squares were cut out before it was bent up to form an open box. Similarly, the concepts of a hemisphere, cylinder and inscription were perfectly explained according to what the task requires. This portrays what is in his mind and he was able to put it in writing. However, the interviewee had some difficulty reaching a consensus between what a sector looks like and the actual concept of a sector (concept-definition and concept-image). The only setback shown by the interviewee in this task was the inability to draw with correct properties, the 
diagram of a sector, as shown in figure 1. Below is the reproduced diagram of what W-2 drew to represent a sector:

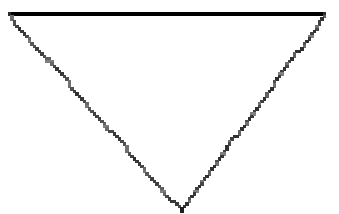

Fig. 1

On the other hand, misunderstanding the question base on the knowledge of basic geometric diagrams poses some problems among low achievers on this task. To illustrate this deficiency, T-20 claimed that "I didn't understand what the question says. All that I knew was circular cylinder and the rest, I didn't know...such as hemisphere, inscription". What provoked this claim was her insufficient knowledge of basic geometric shapes. In a similar vein, V-2 demonstrated the absence of basic geometric concepts in the course of our interaction during the interview. The excerpt from the interview is given below:

$\mathrm{RCH}$ : Let us start with question $1 \mathrm{~b}$, even though you got 1a right, tell me how you solved it?

V-2: $\quad$ Uhm....right circular cylinder...I don't know what that is...I have never heard of that. I don't know if it is like a cylinder or it has a right angle in it. But I know it is inscribed in a hemisphere.

$\mathrm{RCH}$ : And you think this is a hemisphere?

V-2: Yeah...probably wrong (laughter).

$\mathrm{RCH}: \quad$ Can you give an example of a hemisphere in real life?

V-2: Just like the earth is circle...I don't know...I mean...

$\mathrm{RCH}$ : You are not sure of the concept of a hemisphere?

V-2: Yes.

$\mathrm{RCH}$ : How about question 1c.

V-2: I have probably didn't know how to start.

$\mathrm{RCH}: \quad$ Okay, but you have a cone right?

V-2: $\quad$ Uhm...I got it from here (pointing at diagram from question three).

$\mathrm{RCH}$ : What is a sector? Did you know what a sector is?

V-2: No.

$\mathrm{RCH}$ : Can you recall a situation where you learned about a sector?

V-2: I may probably do...I don't remember that.

$\mathrm{V}-2$ is another participant in the study who clearly shows some deficiency or absence of facts on basic geometric diagrams. For example, the concept of hemisphere was illusive even though there was an attempt to make a connection with real life, which itself was hazy as illustrated in Figure 2. From the interviewee's test script (see Figure 2), a cylinder was inscribed in a circle (assuming circle to be a sphere), but the task required a hemisphere. 


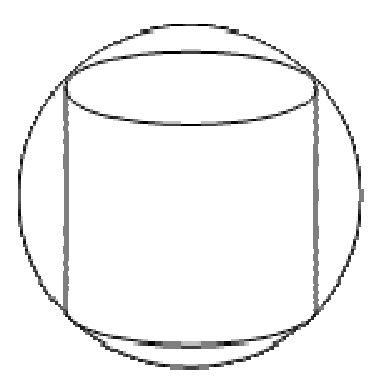

Fig. 2

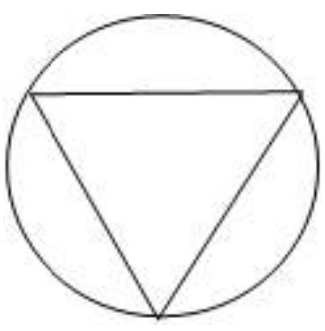

Fig. 3

So, according to the interviewee's understanding, as indicated by the test script; a sphere is considered to be a hemisphere. In the course of the interview, the student was not sure whether the solution given, i.e. Fig. 2, was correct. On a related issue, the concept of a sector again poses a setback to the interviewee. Evidently, from the student's written work (Fig. 3), it was revealed that the student was unable to cut a sector from a circle as required by the task.

Summarizing the qualitative results of the first research question, most students showed a good understanding of the basic geometric shapes that are represented in word problems, even though there are some that illustrated lack of this knowledge. There were also indications that visualization ability was a problem among the students, most especially moving from two-dimensional to threedimensional diagrams. Misunderstanding or unfamiliarity with the concepts of hemisphere and inscription were a hurdle among low achieving-students in this task. Other revelations from the qualitative results are that few claimed misunderstanding of the question, which was clearly grounded on their insufficient facts on basic geometric concepts. Overall, establishing a relation between concept-definition and concept-image as it is reflected in the minds of individuals (Hershkowitz), what, (Vinner, 1983) called the product of concept- formation-process in the mind, was proved to be difficult.

Research question 2): Are the students able to label given geometric diagrams using variables?

These research questions examine whether students could label a constructed diagram using one variable explicitly, calculate dimensions, or a non-canonical dimension using variables. The focus is to assess students' conceptual understanding of variables and their applications to new, independent and appropriate situations. There were three test questions designed to assess these targeted qualities. The concepts evaluated include expressing dimensions using a single variable via the terms "twice as" or "a third of"; the concept of dimension itself; establishing the relationship between $L$, (length of the rectangular piece of metal) and $2 \pi \mathrm{r}$, (circumference of the top of the resultant cylinder). Another conceptual hurdle expected is labelling the sides of a non-routine and a non-canonical geometric diagram using only four variables.

Information derived from the quantitative portion of the study indicated that the mean score for this test item was 6.34 points, with 7 points being the most frequent score. The quantitative data from this research question can be divided into two categories, using the average score as a relative benchmark. Using the average as a cut off point, 63 students (40.7\%) scored between 0 to 6 points; whereas $92(59.3 \%)$ achieved a score between 7 to 9 . A general inference drawn from examination of the qualitative data was that the interviewees vary in their capability and a majority of the students found it difficult to use geometric (mathematical) language, for example dimensions of a cylinder. Moreover, establishing the relationship between the length of a rectangle and its symbolic representation in the new transformed situation (cylinder), as well as picking four variables (two each horizontally and vertically) for the labeling of a non-canonical geometric figure, remained a very difficult task. Among the successful ones who came to terms with concept of dimensions of a cylinder 
was V-15. He/She was able to set up a relationship between length $(L)$, of a rectangular piece of metal and the perimeter (circumference of the top of the cylinder), before expressing the required dimension, i.e. $r$ in terms of $L$. Below is the excerpt reproduced manuscript of V-15:

$$
\begin{aligned}
& P=L \\
& \quad P=\pi 2 \mathrm{r} \\
& \quad \therefore r=\frac{L}{2 \pi}
\end{aligned}
$$

In the (59.3\%) category of students, a majority of them including L-7, J-23, and S-3 found it difficult to interpret the question. L-7 clearly had no idea what the dimensions of a cylinder are at the beginning of interview, but later picked it up when the concept was associated with some familiar situation. On the other hand, J-23 and S-3 had a similar struggle even when a familiar situation was cited, but still they could not comprehend it. Below was how the conversation went with J-23:

$\mathrm{RCH}$ : When you transform the rectangle into a cylinder, you generate a circumference here. J-23: Right.

$\mathrm{RCH}$ : And then you generate a height, so what are the dimensions of a cylinder in your new object?

J-23: $\quad \ldots$ (no response).

$\mathrm{RCH}$ : Lets look at here, what are the dimensions of a rectangle?

J-23: $\quad$ Length times width.

$\mathrm{RCH}$ : Then what are the dimensions of the cylinder?

J-23: Aaa...are you going to take into account volume or not necessarily? Is that what you are asking?

RCH: No...no. The dimensions....you know what the dimensions means?

J-23: Yeah....are the ...would the circumference, plus the width...

The concept of dimension as a mathematical (geometric) language caused some hardship for J-23 in doing calculating labeling. Calculating labeling tasks requires setting up a relationship between two expressions and a variable of interest which is expressed in terms of other(s). For example, using the relation (equation) $L=2 \pi r$ to express $r$ in terms of $L$. The student showed misunderstanding of the meaning of dimensions itself as well as its application to the specified situation. The student tried as much as possible to guess what the meaning of dimension was by making a statement and asking if the statement referred to dimension, as well as by thinking of dimension in the sense of onedimensional, two-dimensional, three-dimensional geometric figures. This is an innovation to cover up for the difficulty experienced as well as an effort to fill-in the gaps. Two separate situations like ". . . take into account volume or not necessarily?" and ". . would the circumference" show these dual novelties of "cover-up and fill-in" effort. In the task that involves the non-canonical geometric diagram, V-2 demonstrated a good understanding of the relationship between horizontal and vertical sides of the figure and was able to use it successfully. My interaction with V-2 follows:

RCH: Okay...lets look at last part of 2c...can you explain how you arrived at your solution?

$\mathrm{V}-2$ : $\quad$ Because this is $\mathrm{L}$ and if you subtract that, you got that small part.

$\mathrm{RCH}$ : So you are able to relate the longer distance with these smaller ones?

V-2: Yes.

In conclusion, students' inability to fully grasp the concept of dimension from geometric perspectives with particular reference to a cylinder leads to misunderstanding what the task requires and consequently, making mistakes in the solution. Similarly, non-familiarity (lack of connection) with the concepts again poses some hindrance to the success of this task. The overall adverse effect of this 
shortcoming was that students are unable to express $r$ in terms of other variables. To a certain extent, some cannot even correctly interpret the statement "express $r$ in terms of $L$ ". This is a clear deficiency in students' mathematical language. The non-canonical task was completed relatively well with a majority of the students establishing a good relationship between the sides (longer and shorter horizontal and vertical lines) of the geometric figure. It can be inferred from this activity that a good understanding and usage of the concepts of variables was well accomplished. The explicit labelling activity involving the use of variables was also fully achieved as a significant portion of the students were successful.

Research question 3): Are the students able to associate given geometric diagrams with their corresponding geometric formula(s)?

The target was to assess how students recognize and associate geometric diagrams with their formulas. They are expected to match geometric formulas with geometric diagrams from the pool of formulas and geometric diagrams. The test instrument contains six geometric diagrams (two and three-dimensional), and twelve formulas in the pool. Both formulas and diagrams are arranged randomly. Although some formulas may be more or less familiar than others, the knowledge of formulas and their relationship with geometric diagrams is factual information. The items explored whether students experienced some difficulty finding an appropriate match as well as whether they could distinguish between two-dimensional and three-dimensional diagrams. Moreover, the items examined students' capability in differentiating the concept of area and surface area with particular reference to two-and three-dimensions. Statistical results from quantitative data showed an average score of 4.7 points out of a maximum 6 points possible, which incidentally, is the most frequent score. The samples were grouped into two using the mean as a cut-off point. The first group had a score range of 0 to 4.5 points with 61 students $(39.3 \%$ ); while the second group had a score range of 5 to 6 points with 94 students $(60.6 \%)$. Further qualitative analyses of these groups were given below.

Generally, students used the process of elimination to associate appropriate geometric diagrams with their formulas. This is called use of strategic knowledge in a negative way. Strategic knowledge refers to knowing when to use a rule. But the process of elimination can be used even if the rules are not well known. The group that scores $(0-4.5$ points $)$ used non-conceptual processes, leading to circumstances where two-dimensional figures ended-up with a volume formula or a threedimensional diagram obtained an area formula instead of surface area formula. A good example of the use of non-conceptual process of elimination emerges from my interaction with T-20 and our conversation went as follows:

$\mathrm{RCH}$ : Okay, almost everything here is perfect (question 3) except amm...what is this? a rectangle right? The first diagram here is a rectangle right, so here you say volume, does a rectangle have a volume?

T-20: (Laughter)......no.

$\mathrm{RCH}$ : So what happens?

T-20: I have no idea.

$\mathrm{RCH}$ : You just probably write.

T-20: Because I was just doing the process of elimination. I don't know why I am doing this.

$\mathrm{RCH}$ : Here you have a box and left it blank, probably you want to write it here but you didn't. Since you follow the process of elimination, can you describe how you get the volume of a cylinder?

T-20: Amm...Okay...I didn't know ...but I knew this is a circle... 
Despite the fact that T-20 succeeded in associating the given formulas with their appropriate diagram, the procedures followed were non-strategic. Similar characteristics were shown by L-7 and V-2. The strategic use of the process of elimination included a good knowledge of and/or familiarity with basic geometric diagrams and their formulas. $\mathrm{J}-23$ and $\mathrm{W}-2$ used this approach and were able to successfully complete the task. Specifically, J-23 separated or grouped two-dimensional geometric diagrams from the three-dimensional ones before associating them with their appropriate formulas. Below gives an account of how our interaction went:

$\mathrm{RCH}$ : Talk to me about your understanding/misunderstanding of associating geometric diagrams with appropriate formula?

J-23: So following each formula with it figure...for area...perimeter...okay. This...this...this and this (circling the $3-\mathrm{D}$ ) have surface areas...they are the only 3 D shapes.

$\mathrm{RCH}$ : Okay.

J-23: $\quad$...and like I cross them out, and I use ...for some of them I use process of elimination. But for the circle I knew...the area and the perimeter... and for the rectangle I knew the area and perimeter. Then, from there...the cube was the only one you can use $L$, because the cube all the sides do the same. The only way you can do length times width times height, all of them be the same. So that the way you do the cube... and then for it surface area, there is six sides and each side is $L$ times $L$ to get the area of it and then times six, because there are six of them. For the rectangle (pointing at rectangular box), I knew that the area (volume, added mine) is length times width times height. For the surface area is the same thing, two of the length times height (2lh), two of the width times height (2wh), and two of length times width (2lw). The cone...cone and cylinder ....am...I actually I don't remember how I got those, only because.....let me think (silently). I won't say I knew this was the volume because we just done that...in class we just went over about the volume of the cylinder.

RCH: Okay.

J-23: So I knew the volume was this (pointing at the formula). This has to be the volume by default because there are no other volumes. ( $\mathrm{RCH}$ interject with laughter). And so...

$\mathrm{RCH}$ : So you mean by default....since there are no any other options, this has to be the volume?

J-23: Right,...this has to be the volume.

RCH: You have already eliminated.....

J-23: Three out of four....and then amm...let see ...this is the surface area that seems to go with...(long silence) this I guess ....because...I think about that, but this...if actually this work together ...then surface area would be smaller than the volume .....that is correct. Am so...I choose .....

$\mathrm{RCH}: \quad \pi \mathrm{rL} \ldots .$.

J-23: Yes....so the cone and the cylinder had a little bit of trouble and it was more of a kind of process of elimination ... actually like figuring it out. And because this was two and two, this would be one and the bottom would be one, this side of it and the back side of it would be one.

The evidence indicates that the student has the ability to create ways of handling tasks that require the matching of concepts, as well as using previous experiences that are relevant. This shows J-23 is resourceful; but certainly, J-23 lacked knowledge of the formulas for the cone and cylinder. The formulas were found by elimination, since all other options are gone. 
In summing up the qualitative results, students experienced some setbacks finding suitable matches between formulas and diagrams, and distinguishing between two-dimensional and threedimensional diagrams, whereas others efficiently did the task. The hurdles encountered were grounded on the students' unfamiliarity with geometric formulas and difficulty recognizing some shapes by name (concept definition and concept image). Overall, process of elimination was used by many students as strategic knowledge, but it was used in a negative way. Despite the fact that some knew the formulas as well as the geometric diagrams, finding an appropriate match without the use of process of elimination was hard. Another important finding from the study was that students were unable to establish proper relationships between concept-definition and concept-image with respect to area and surface area of two and three dimensional geometric diagrams.

Research question 4): What kind of geometric ability do students possess in solving ACOP completely?

This research question assessed student's geometric and basic differentiation skills in ACOP solutions. Specifically, ACOP solution process would show the exact point of breakdown that is either geometric or basic differentiation skills, i.e. fault lines. There are three tasks designed to address this research question. The tasks were different from each other with a certain degree of conceptual variability. Overall, the conceptual difficulties include finding the dimensions that are expressed in a single variable, area formula, and the nature of poster problem. Others consist of identifying a trapezoidal structure from a sketch of the word problem, labelling with new variables, and deriving the required equation in terms of variable of interest. Structural inabilities might be apparent in calculating the derivatives of the derived trigonometric equation, use of appropriate identities and finding the critical points.

Results from the quantitative portion indicate that the mean score of this task is 1.83 with 9 possible points. Overall sample performance on the task based on this output was that $106,(86.4 \%)$ scored 0 to 2 points and $49(31.6 \%)$ scored 3 to 6 points. Drawing a remark base on these facts indicates that a significant percentage of the sample had performed poorly in complete ACOP tasks. Qualitative results were used to support this claim. L-7 succeeded in visualizing, drawing and labelling sides of the required geometric diagram, but setting up a relationship between numerical and algebraic volume and deriving appropriate formula were obscured. A similar partial success story was recorded with $\mathrm{W}-2$ and J-37, where setting up the equation to be optimized was accomplished, but getting the derivative was not successful. Below is the reproduced work of W-2:

$$
\begin{gathered}
x y=384 \mathrm{~cm}^{2} \quad(y+12)(x+8) \\
x=\frac{384}{y} a^{\prime}=\frac{384}{y}+8+y+12 \\
-20=\frac{384}{y}+y \\
y^{2}+20 y+384 \\
y^{2}+20 y=384
\end{gathered}
$$

Clearly, there is a structural failure in applying the product rule to differentiate the derived equation. Moreover, W-2 was unable to recognize the quadratic structure in the assumed correct first derivative, hence leading to an un-strategic solution approach. Finally, W-2 can't visualize the rain gutter from the question. In the same way, S-3 had difficulty in visualizing and drawing the poster and rain gutter, but succeeded in expressing the relationship between numerical and algebraic volumes. In a related development, V-2 couldn't visualize and draw the poster and rain gutter as well as labeling the diagrams. In fact, interpretation of mathematical language "material required to construct", i.e. surface area, was hard. The student was undecided on what to use between area and volume formulas. Among the relative success stories in this section was J-37. The student clearly 
visualizes the rain gutter, label it using appropriate variables, derived the required equation and calculate its derivative. However, the use of less simple variables defined in trigonometric functions lead to incorrect results.

Summarizing qualitative data for the last research question revealed the difficulties experienced by the students and they include, visualization, drawing, and in some instances labeling (when it was not apparent), as well as application of the appropriate differentiation rule (power, product or quotient rule). Moreover, interpreting mathematical language such as "least material", making a connection between algebra and trigonometry functions (structural and procedural) as well as fluent use of trigonometric identities appeared to be causing some nuisance.

\section{Conclusion, Discussion and Recommendation}

Generally, students performed well in the isolated tasks of constructing and labeling a diagram. Results from the quantitative data indicated a high mean score and this was equally supported by the qualitative outcome. Most students showed a good understanding of the basic geometric shapes that were represented in word problems, perceptual apprehension,(Duval, 1995), cited by (Panoura \& Gagatsis, 2009); although a few illustrated lack of this ability. For example, the unsuccessful ones in this activity were hindered by their low level ability to transform two-dimensional to threedimensional objects, and vice versa, a result that was consistent with Mitchelmore (1980) cited by Hershkowitz (1990). Similarly, Koc, Y., et al and van Hiele (1986) call this "inability to reach descriptive level of geometry". Moreover, according to Hershkowitz, three factors influenced the description and interpretation of three-dimensional drawings. These are: culture, experience and familiarity. It was evident from the qualitative results that lack of familiarity and in-experience plays a major role in hindering students in these activities. A good example is the task of transforming a sector of a circle into a cone. Students interviewed acknowledged that, either they didn't know what a sector was (in-experience) or they couldn't fully describe what a sector looks like (familiarity). A cultural factor may not be a significant factor in obstructing success in this situation because there are so many objects that could be used to express basic geometric shapes in their immediate cultural environment. Overall, the lingering difficulties that thwarted these few students' success in these tasks were clearly grounded in their insufficient knowledge of fundamental geometric concepts.

The deficiencies in students' mathematical language had hindered the calculating-labeling task, but for non-standard shapes created for the test, explicit labeling was relatively successful. One major constraint faced by students in the non-standard labeling task was their inability to recognize the functional relations among related elements of the diagram. Establishing this relationship was a serious problem among the unsuccessful students. The calculating-labeling task was hindered by a geometric language deficiency. Specifically, students had demonstrated a lack of familiarity and understanding with the concept of dimensions of a cylinder. This language deficit had totally obstructed a majority of these students who failed on these items from accomplishing what the task requires. Other findings revealed that using expressions like 'in terms of these variables' seems confusing to some. All these were rooted as a result of the shortage of students' mathematical (geometric) language. In a dissimilar trend, students had demonstrated familiarity with factual knowledge of geometric formulas, but blind use of the process of elimination and overgeneralizations, in addition to misinterpreting the question's instruction, prevented some students from achieving what was required by tasks.

Optimizations solution processes started with the visualization of geometric diagrams described in word problems. It was clear that students were unable to visualize geometrically. Failure to put down the visual image e.g. rain gutter, followed by the inability to construct an additional geometric modification to complete the diagram, had led to a chain reaction collapse (geometric, or basic 
differentiation) in the solution process. Another source of breakdown is labeling complex geometric diagrams in terms of information given. Consequently, there is lack of interaction between sequential, discursive and operative apprehension (Duval, 2006; Panaoura \& Gagatsis). The concept of a variable was well understood, but a non-strategic solution process led to the derivation of a complicated equation. Another step toward the solution of the optimization problem requires equating the first derivative of a constructed equation to zero and solve the variable of interest algebraically. Finding meaning in a statement such as $\frac{d y}{d x}=0$ was already shown to be out of reach, i.e. students couldn't explain why it was done.

There are a lot of educational implications that could be derived from this study. The discussion of results was centred on three major components (geometry, visualization, and basic differentiation skills) and how each influences the solution of the optimization problem. According to Hershkowitz, "visualization generally refers to the ability to represent, transform, generate, communicate, and reflect on visual information" (p.75). She further cited Bishop (1989), who claimed that, "visualization is important not only for its own sake but also because the type of mental processes involved are necessary for, and can transfer to, other areas of mathematics" (p.76), examples of geometry and applied calculus.

Indeed, the results from both quantitative and qualitative portions revealed that to a certain degree, students have an understanding of basic geometric concepts (isolated cases), but integrating those capabilities proved difficult in the solution of optimization problem. The results exposed that students had faced some difficulty creating a concept-image from concept-definition; Vinner (1983) cited by Hershkowitz, that is, they were unable to interpret and show some understanding of the concept presented in word problems and translated them into concept images. According to Hershkowitz, concept is derived from its mathematical definition and has attributes. Those attributes were used to separate examples from non-examples. The educational implications of these findings for the school curriculum and college teaching were to adapt geometry teaching methods that emphasize establishing a good relationship between concept-definitions and concept images. It was revealed that visualization-skills involving interpreting figural information is trainable, (Bishop). Based on this, it is imperative for teachers of geometry to teach geometry (visualization) and other aspects of it from conceptual perspectives and avoid rote learning which is highly likely to be forgettable. Reiterating this position, Jones (2002) opines that, "effective learning approaches encourage students to recognize connections between different ways of representing geometric ideas and between geometry and other areas of mathematics ... it would help students retain knowledge and skills and enable them to approach new geometrical problems ( isolated or non - isolated tasks) with confidence",(p. 132 - 133). Incorporating this valuable opinion into applied calculus classes would be of utmost importance.

\section{References}

Arcavi, A. (2003). The role of visual representations in the learning of mathematics.Educational Studies in Mathematics, 52, 215241.

Barret, J. E., \& Clements, D. H. (2003). Qualifying length: fourth-grade children's developing abstractions for measures of linear quantity. Cognition and Instruction, 21(4), $475-520$.

Battista, M. (2007).The development of geometric and spatial thinking.In F.K. Lester(Ed), Second handbook of research on mathematics teaching and learning (843 - 908). Greenwich, CT: Information Age Publishing.

Battista, M.T. (2001a). A research-based perspective on teaching school geometry. In J. Brophy (Ed), Advances in research on teaching: Subject-specific instructional methods and activities (pp. 145 -185). New York: JAI Press.

Bishop, A. J. (1989). A Review of research on visualization in mathematics education.Focus on Learning Problems in Mathematics, $11(1 \& 2), 7-16$.

Bremigan, E. G. (2005).An analysis of Diagram Modification and Construction in Students' Solution to Applied Calculus Problem.Journal for research in Mathematics Education, 36 (3), 248 - 277.

Brijlall, D., \&Ndlovu, Z. (2013). High school learners' mental construction during solving optimization problem in Calculus: a South African case study. South African Journal of Education; 33(2). 
Burton, M. B. (1989). The Effect of Prior Calculus Experience on "Introductory" College Calculus. The American Mathematical Monthly, 96(4), $350-354$.

Chappell, M. F., \& Thompson, D. R. (1999).Perimeter or Area? Which measure is it? Mathematic Teaching in the Middle School, $5(1), 20-23$.

Clements, D. H.., Battista, M. T., Sarama, J., Swaminathan, S., \&McMillens, S. (1997). Students' development of length concepts in a Logo-based unit on geometric paths.Journal for Research in Mathematics Education, 28(1), 70 - 95.

Davis, R. B. (1986). Calculus at University High School. In Douglas, R.G. (ed). Toward a Lean and Lively Calculus.MAA Notes Number 6, Mathematical Association of America.

Donaldson, M. (1963).A study of children's thinking (pp.183-185). Tavistock publications: London.

Dreyfus, T. (1991).On the status of visual reasoning in mathematics and mathematics education.In F. Furinghetti (Ed.), Proceedings of the $15^{\text {th }}$ Conference of the International Group for the Psychology of Mathematics Education (Vol. 1, pp. 3348).Assisi, Italy: PME.

Duval, R. (1995). Geometrical pictures: kinds of representation and specific processings. In R. Sutherland \& J. Mason (Eds), Exploring Mental Imagery with Computers in Mathematics Educations (pp. 142 - 157). Berlin: Springer-Verlag.

Duval, R. (2006).A cognitive analysis of problems of comprehension in the learning of mathematics.Educational Studies in Mathematics, 61, 103-131.

Ferrini-Mundy, J., \& Graham, K. G. (1991). An Overview of the Calculus Curriculum Reform Effort: Issues for Learning, Teaching, and Curriculum Development. The American Mathematical Monthly, 98(7), 627 - 635.

Gutrierrez, A. (1997). Visualization in 3 - Dimensional Geometry: in search of a Framework. Retrievd from: http://www.uv.es/angel.gutierrez/archivos1/textospdf/Gut96c.pdf, on 16/1/14.

Hershkowitz, R., et al (1990). Psychological Aspects of Learning Geometry (Nesher, P., \& Kilpatrick, J, Eds).Mathematics and Cognition: A Research Synthesis by the International Group for the Psychology of Mathematics Education. (p. 69 - 95). Cambridge: Cambridge University Press.

Hohenwarter, M. \& Jones, K., (2007). Ways of Linking Geometry and Algebra: The Case of Geogebra. In D. Kuchemann (Ed), Proceedings of the British Society for Research into Learning Mathematics, 27(3), 126 - 131.

Hurley, J. F., Koehn, U., \&Ganter, S. L. (1999). Effects of Calculus reform: Local and National. The American Mathematical Monthly, 109(9), $800-811$.

Jones, K. (2002). Issues in the Teaching and Learning of Geometry. In Linda Haggarty, (Ed), Aspects of Teaching Secondary Mathematics: perspectives on practice. London: RoutledgeFalmer. (p. 121 - 139).

Jones, K. (2000). Teacher Knowledge and Professional Development in Geometry. Proceedings of the British Society for Research into Learning Mathematics, 20(3), 109 - 114.

Jones, K. \& Mooney, C. (2003).Making space for geometry in primary mathematics. In I. Thompson (Ed), Enhancing primary mathematics teaching. London: open press, $3-15$.

Koç, Yusuf, et al. (n.d) "An Investigation on Students' degree of Acquisition Related to Van Hiele Level of Geometric Reasoning: A Case of 6-8 Th Graders in Turkey." http://cerme8.metu.edu.tr/wgpapers/WG4/WG4_Koc.pdf

Martin, G., \&Structchens, M. (2000).Geometry and measurement.In E. A. Silver \& P. A. Kenny (Eds), Results from the seventh mathematics assessment of the National Assessment of Educational Progress (p. 193 - 234). Reston, VA: NCTM.

Marchett, P., et al. (2005). Comparing Perimeters and Areas Childrens' Pre-conceptions and spontaneous procedures.CERME 4. $766-775$.

Matta, C, Feinberg G, Cardetti, F. (2012).Exploring Learning difficulties in multivariable Calculus. Retrieved from: http://mathreu.uconn.edu/Math\%20ED\%20REU\%20Poster.pdf on 29/09/2015

Muzangwa, J., \&Chifamba, P. (2012).Analysis of Errors and Misconceptions in the Learning of Calculus by undergraduate students.ActaDidacticaNapocensia, Vol. 5 (2).

National Council of Teachers of Mathematics, (2000).Curriculum and Evaluation Standards for School Mathematics. Reston, VA: NCTM.

Nunes, T., Light, P., \& Mason, J. (1993). Tools for thought: the measurement of length and area. Learning and Instruction, 3, 39 54.

Panaoura, G., \&Gagatsis, A. (2009).The Geometrical Reasoning of Primary and Secondary School students.Proceedings of CERME 6, Working Group 5. January 28th - February 15t, 2009. Lyon France $\odot$ INRP 2010.

Pesek, D. D., \&Kirshner, D. (2000).Interference of instrumental instruction in subsequent relational learning.Journal for Research in Mathematics Education, 31(5), $524-540$.

Pittalis, M., \& Christou, C. (2010).Types of reasoning in 3D geometry thinking and their relation with spatial ability.Educational Studies in Mathematics, 75(2), 191-212.

Presmeg, N. C., \& Balderas-Canas, P. E. (n.d.). Graduate Students' Visualizations in Two Rate of Change Problems. Retrieved from: http://www.academia.edu/6665583/Graduate_Students_Visualizations_in_two_Rate_of_Change_ Problems.

Stylianou, D. A., \& Pitta-Pantazi, D. (2002). Visualization and High Achievement in Mathematics: A Critical Look at Successful Visualization Strategies. In Fernando Hitt (ed). Representations and Mathematics Visualization, North American Chapter of the International Group for the Psychology of Mathematics Education, Working group (1998 - 2002).

vanHiele, P.M. (1986). Structure and insight: A theory of mathematics education. Orlando, Fla.: Academic Press

Vinner, S. (1983).Concept definition, concept image and the notion of function.International Journal of mathematical Education in Science and Technology, 14, 239-305. 
Woodward, E., \& Byrd, F.)1983). Area: Included topic, neglected concept. School Science and Mathematics, 83(4), 343 -347.

Yasin, S., \&Enver, T. (2007). Students' Difficulties with Application of Definite Integration. EducațiaMatematică Vol. 3, Nr. 1-2, $15-27$

Young, G. S. (1986). Present Problems and Future Prospects. In Steen, L.A. (ed). Calculus for a New Century. Mathematical Association of America Notes Number 8, 172 - 175.

Zimmermann, W., \& Cunningham, S., (1991).Visualization in Teaching and Learning Mathematics. MAA Notes No. 19. Washington D.C.: Mathematical Association of America.

\section{Acknowledgement}

The content of this paper is an extract from my unpublished dissertation at Louisiana State University, Baton Rouge, 2008. I can confirm to you that it was not publish elsewhere.

I would also like to acknowledge the contribution of Mr Ludwig Beni, English Language department, Jubail University College, male branch, KSA, for his time in editing the paper. 